\title{
Familial Cold Autoinflammatory Syndrome
}

National Cancer Institute

\section{Source}

National Cancer Institute. Familial Cold Autoinflammatory Syndrome. NCI Thesaurus.

Code C119053.

An autoinflammatory disease caused by mutations in the NLRP3 gene which encodes

cryopyrin. It is characterized by short episodes of fever, rash, and arthralgia after exposure to cold or rapid decrease in temperature. 